\title{
Improving health care efficiency through the integration of a physician assistant into an infectious diseases consult service at a large urban community hospital
}

\author{
Melissa Decloe MSc CCPA ${ }^{1,2}$, Janine McCready MD FRCPC ${ }^{1,3}$, \\ James Downey MD FRCPC PhD ${ }^{1,3}$, Jeff Powis MD FRCPC $\mathrm{MSc}^{1,3}$
}

\begin{abstract}
M Decloe, J McCready, J Downey, J Powis. Improving health care efficiency through the integration of a physician assistant into an infectious diseases consult service at a large urban community hospital. Can J Infect Dis Med Microbiol 2015;26(3):130-132.
\end{abstract}

BACKGROUND: Physician assistants (PAs) have recently been introduced into the Canadian health care system in some provinces; however, there are little data demonstrating their impact.

METHODS: A retrospective case-control study was conducted between January 2010 and December 2013. Length of stay (LOS) and mortality were examined in the infectious diseases consult service (IDCS) compared with hospital-wide controls. The two-year period before the introduction of the PA to the IDCS of a large urban community hospital in Canada (2010 to 2011) was compared with the two-year period following the introduction of the PA (2012 to 2013). RESULTS: Following the introduction of a PA to the IDCS, there was a decrease in time to consultation from $21.4 \mathrm{~h}$ to $14.3 \mathrm{~h}$ $(\mathrm{P}<0.0001)$. LOS was significantly decreased among IDCS patients by 3.6 days more than that seen in matched hospital-wide controls $(\mathrm{P}=0.0001)$. Mortality did not significantly change after PA introduction in either cases or controls.

DISCUSSION/CONCLUSION: PAs can improve health efficiencies in the Canadian health care setting, leading to reduction in LOS.

Key Words: Length of stay; Mortality; Physician assistant

\author{
Améliorer l'efficacité des soins de santé par \\ l'intégration d'un auxiliaire médical au service \\ de consultations infectieuses d'un grand hôpital \\ général en milieu urbain
}

HISTORIQUE : Les auxiliaires médicaux (AM) ont récemment été intégrés au système de santé de certaines provinces canadiennes, mais on possède peu de données pour en évaluer les répercussions.

MÉTHODOLOGIE : Les chercheurs ont réalisé une étude rétrospective cas-témoins entre janvier 2010 et décembre 2013. Ils ont comparé la durée de séjour (DdS) et la mortalité au service de consultation en infectiologie (SCI) à celles de sujets témoins de tout l'hôpital. Ils ont comparé la période de deux ans avant l'arrivée des AM au SCI d'un grand hôpital général urbain du Canada (2010 et 2011) à la période de deux ans suivant leur arrivée (2012 et 2013).

RÉSULTATS : Après l'arrivée des AM au SCI, les chercheurs ont constaté une diminution du délai avant la consultation, qui est passé de 21,4 h à 14,3 h $(\mathrm{P}<0,0001)$. La DdS a régressé d'au moins 3,6 jours chez les patients du SCI par rapport à celle des sujets témoins de l'ensemble de l'hôpital $(\mathrm{P}=0,0001)$. La mortalité ne changeait pas de manière significative après l'arrivée des $\mathrm{AM}$, que ce soit pour les cas ou les sujets-témoins.

INTERPRÉTATION : Les AM peuvent améliorer l'efficacité du milieu canadien de la santé et réduire la DdS.

Dhysician assistants (PAs) are academically trained, dependent health 1 care providers who work in various clinical settings to support the clinical duties of the supervising physician (1). They have been practicing in Canada since the 1960s in the armed forces and have recently been introduced into civilian practice in some provinces (2). Manitoba and Ontario have established PA training programs (2) and Alberta has recently introduced a pilot project to implement PAs (3). The impact of PAs in other jurisdictions, particularly in the United States, has been well studied and extrapolated to inform the introduction of PAs in Canada. The generalizability of the United States literature is limited by the substantial differences between health care systems; specifically, the significant differences in health care funding. Given that the profession remains in its infancy in Canada, research has only begun to emerge examining the integration of PAs in Canadian practice (4). To date, the majority of Canadian studies have aimed to describe the role of PAs in Canada $(5-8)$ as well as physician and patient attitudes toward PAs (9-12). Three studies were identified that aimed to evaluate outcomes relating to implementing a PA into Canadian practice; however, all were limited by their lack of a control group (13-15). To date, no study has subspeciality in Canada. Therefore, we aimed to evaluate the impact of integrating a PA into a medicine subspeciality on health care efficiency and patient outcomes.

\section{METHODS}

Starting in November 2011, the Infectious Diseases Consult Service (IDCS) at Toronto East General Hospital (Toronto, Ontario), a 515. bed urban community hospital, introduced a PA with assistance from a Career Start Grant for Physician Assistant Graduates through Health Force Ontario (16). The PA was a Canadian Certified Physician Assistant and had completed a Canadian training program in 2011. Infectious diseases (ID)-specific training consisted of two weeks in the offsite microbiology laboratory as well as ongoing one-onone mentorship with the ID specialists during daily case reviews.

A retrospective case-control study was conducted between January 2010 and December 2013. The two-year period before the introduction evaluated the impact of integrating a PA into a hospital-based medicine

${ }^{1}$ Division of Infectious Diseases, Department of Medicine, Toronto East General Hospital, Toronto; ${ }^{2}$ Physician Assistant Education Program, McMaster University, Hamilton; ${ }^{3}$ Department of Medicine, University of Toronto, Toronto, Ontario

Correspondence: Dr Jeff Powis, Division of Infectious Diseases, Department of Medicine, Toronto East General Hospital, Toronto, Ontario. Telephone 416-469-6252, fax 416-469-6253, e-mail jpowi@tegh.on.ca 
TABLE 1

Baseline characteristics of the infectious diseases cohort (cases) and hospital-wide cohort (controls)

\begin{tabular}{lcc}
\hline Variable & Cases $(\mathbf{n}=3386)$ & Controls $(\mathbf{n = 1 3 , 4 9 3 )}$ \\
\hline Age, years, mean \pm SD & $65.9 \pm 18.4$ & $65.9 \pm 18.3$ \\
Sex & & \\
$\quad$ Male & $1735(51.2)$ & $6899(51.1)$ \\
$\quad$ Female & $1651(48.8)$ & $6594(48.9)$ \\
Medical diagnosis partition & $2677(79.1)$ & $10,685(79.2)$ \\
Study period & & \\
$\quad$ Before physician assistant & $1547(45.7)$ & $6160(45.7)$ \\
After physician assistant & $1839(54.3)$ & $7333(54.3)$ \\
\hline
\end{tabular}

Data presented as $n$ (\%) unless otherwise indicated

of the PA (2010 to 2011) was compared with the two-year period following the introduction of the PA (2012 to 2013). The primary objective of the present study was to evaluate the impact of PA introduction on inpatient length of stay (LOS) and mortality among IDCS patients, compared with a matched hospital-wide control group. The inclusion of a control group was critical to determine the independent impact of PA introduction to the IDCS from other hospital-wide quality improvement interventions that may have impacted LOS or mortality.

Patients were included if they were seen by the IDCS during their inpatient stay during the workweek when the PA was working. Patients from the complex continuing care unit, day surgery, mental health and those who were seen in the emergency department but not admitted to hospital were excluded. LOS, mortality and time to consult data were obtained from the hospital electronic medical record. In the LOS and mortality data set, LOS and mortality data were retained for each patient on each unique hospital encounter. The hospital-wide data for the control cohort were obtained from the electronic medical records through hospital decision support. Controls were determined from a hospital-wide data set and were matched to cases according to sex (exact), admission date ( \pm 15 days), age ( \pm 5 years) and diagnosis partition (medical or surgical). The diagnosis partition is part of the Canadian Institute for Health Information's Case Mix Groups methodology, which assigns patients into more homogenous groups for determination of complexity and estimated resource use (17). The aim was to match cases to controls with a $1: 4$ ratio $(18,19)$. Age and diagnosis partition were weighted at two times admission date. Duplicate consults were removed from the time to consultation analysis but were retained if requested $>24$ $h$ after the initial consult.

Time to ID consult in the period before the introduction of the ID PA (preintervention) was compared with the period following the introduction of the ID PA (postintervention) with the MannWhitney U test. A matched case-control analysis was conducted for the LOS and the mortality variables. Of the 3386 patient encounters in the case cohort, 3347 were matched to four controls, 30 were matched to three controls, seven were matched to two controls, one was matched to one control and only one case was not matched to any controls. The unmatched case was a 94-year-old male medical patient.

To estimate differences in means and proportions in LOS and mortality between the cases and controls in the preintervention period to the postintervention period, a generalized estimating equation (GEE) was used. For LOS, a linear GEE model was used to account for the continuous nature of the LOS variable. For the mortality analysis, a logistic GEE model was used for the binary nature of the mortality variable. The project was approved by the local research ethics board.

\section{RESULTS}

Following the introduction of the PA to the IDCS, the average time to consult decreased from $21.4 \mathrm{~h}$ to $14.3 \mathrm{~h}(\mathrm{P}<0.0001)$. Characteristics of cases and controls are shown in Table 1.

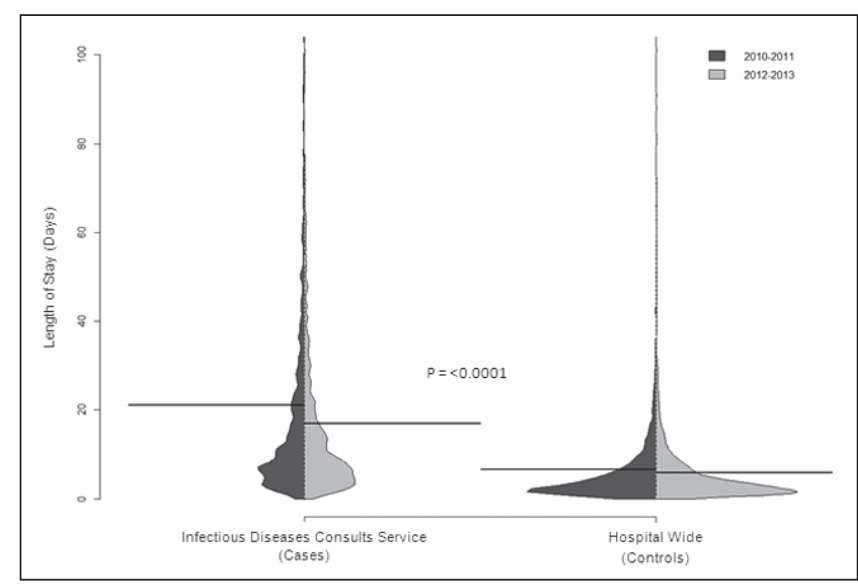

Figure 1) Violin plot illustrating the distribution of length of stay (LOS) in the infectious diseases consult service cohort (cases and the hospital-wide cohort [controls]) pre and post introduction of the infectious diseases physician assistant. The average LOS in each group is illustrated with the bold black line. The differential change in LOS in the cases and the controls is statistically significant $(P<0.0001)$

Among patients seen by the IDCS, there was $21.0 \%$ reduction in LOS, with the average LOS decreasing from 20.5 days to 16.2 days following the introduction of the PA. In the hospital-wide matched cohort, there was a $10.6 \%$ reduction in LOS, with the average LOS decreasing from 6.8 days to 6.1 days from the pre- to postintervention period. There is a statistically significant decrease in the differential change of LOS among patients seen by the IDCS compared with the hospital-wide matches of 3.6 days (95\% CI 1.8 to 5.5 days; $\mathrm{P}=0.0001$ ) (Figure 1).

The proportion of deaths among ID-consulted patients decreased from 0.26 to 0.22 from the pre- to postintervention period; in the hospital-wide cohort, the proportion of deaths decreased from 0.055 to 0.051 during the study period. There was no statistically significant difference in the differential change in the proportion of deaths in the two cohorts $(\mathrm{P}=0.14)$.

\section{INTERPRETATION}

Following the introduction of the PA to the IDCS at Toronto East General Hospital, decreases in both time to consultation and LOS were observed. When LOS in the IDCS cohort was compared with the hospital-wide control cohort, a statistically significant difference in the rate of change of 3.6 days was seen. This suggests that the observed decrease in LOS after introduction of the PA was unique to the IDCS and, therefore, cannot be attributed to any other hospital-wide interventions. Although a trend toward decreasing mortality was observed among the IDCS cohort after introduction of the PA, this did not reach statistical significance.

The significant change in LOS among the ID-consulted cohort in the post-ID PA time periods is likely related to the more rapid time to consultation as well as the fact that the ID PA's primary job responsibility was to the ID consult service. Dedicated ID PA availability likely translated into more frequent follow-up assessments, improved accessibility and facilitated communication to other care providers. Although we did not measure which factors led to the observed reductions in LOS, the PA likely served as a conduit for immediate knowledge translation to consulting services.

Although advancements in the management of ID through new antimicrobials or delivery of current treatments may have been introduced during the study period, none would be anticipated to have such a considerable impact on LOS.

No previous studies have examined LOS in admitted patients in which PAs have been involved in patient care; one previous study examined LOS in the emergency department when PAs were on duty 
and demonstrated similar findings. Ducharme et al (14) examined LOS in the emergency department in a 14-day study period before the introduction of PAs compared with a 14-day study period following the introduction of the PA, and a $30.3 \%$ decrease in LOS in the emergency department was observed when PAs were on duty $(95 \% \mathrm{CI}$ $21.6 \%$ to $39.0 \% ; \mathrm{P}<0.01)$. The findings of this study, however, are limited by the lack of a control group. We are unaware of any literature that evaluated outcomes associated with the introduction of a PA to inpatients, including medicine subspecialities, in the Canadian health care system.

Two studies were identified that conducted cost analyses of using PAs in Canada. Sigurdson (15) conducted a business case analysis that examined PAs employed on a plastic surgery team in Halifax, Nova Scotia and found that a PA could be cost efficient when a double operating room model was used for surgical services. Bohm et al (13) demonstrated similar findings in their study examining a PA employed as a surgical assist in an arthroplasty program in Winnipeg (Manitoba). In their study, PAs were cost neutral with general practitioner surgical assistants, but decreased surgical wait times from 44 weeks in the preintervention time period to 30 weeks postintroduction of the PA (13). Our study did not undertake a financial analysis associated with the decreased LOS in hospital in patients because it was beyond the scope of the current project. However, a decrease in LOS by 3.6 days per patient would undoubtedly be associated with significant cost savings when the cost per day of hospital admission is considered and could form the basis of a business plan to justify employing a PA.

The present study used a matched case-control methodology to examine two years of data in the period before the introduction of the PA and two years of data following the introduction of the PA. This resulted in a large sample size and allowed for the examination of trends over a four-year period, serving to reduce the chance that

\section{REFERENCES}

1. Canadian Association of Physician Assistants - About Physician Assistants. <https://capa-acam.ca/about-pas/> (Accessed May 6, 2014).

2. HFO. Ontario's Physician Assistant Initiative: An Overview. 2009 $<$ www.healthforceontario.ca/UserFiles/file/AHP/Inside/ PA-overview-may-2009-en.pdf> (Accessed April 7, 2014).

3. Alberta Health Services - Physician Assistants. 2014. $<$ www.albertahealthservices.ca/8754.asp > (Accessed April 7, 2014).

4. Hamilton GM, Decloe MD. Reviewing the Evidence for Canada's Physician Assistant Initiative. University of Toronto Medical Journal 2011;88:88-90.

5. Jones IW. Where the Canadian physician assistants are in 2012. JAAPA 2012;25:54.

6. Jones IW, Seo B, Chauhan TS, Buske L. The results of the first Canadian national physician assistant survey. JAAPA 2011;24:63.

7. Jones IW, St-Pierre N. Physician assistants in Canada. JAAPA 2014:27:11-3.

8. Doan Q, Sabhaney V, Kissoon N et al. The role of physician assistants in a pediatric emergency department: A center review and survey. Pediatr Emerg Care 2012;28:783-8.

9. Doan Q, Hooker RS, Wong H, et al. Canadians' willingness to receive care from physician assistants. Cana Fam Physician 2012;58:e459-64.

10. Doan Q, Piteau S, Sheps S, et al. The role of physician assistants in pediatric emergency medicine: The physician's view. CJEM 2013;15:321-9.

11. Taylor MT, Wayne Taylor D, Burrows K, Cunnington J, Lombardi A, Liou M. Qualitative study of employment of physician seasonal variations in patient volumes or other initiatives impacted the findings. One of the largest limitations of our study is that no measure of patient severity or complexity was available for matching. The mortality rate and the average LOS were higher in the IDCS cohort compared with the hospital-wide cohort, demonstrating that the IDCS cohort and the hospital-wide cohort are likely systematically different from one another. More information may have been gained from the mortality analysis if we were able to match cases based on patient complexity or severity. It should be reiterated, however, that the inclusion of the hospital-wide cohort was not for direct comparison of these two very different groups, but rather to provide context for our IDCS analysis. The present study was also limited by its retrospective design; thus, there may be unmeasured confounders biasing our results that were differentially affecting the IDCS patients. Finally, only a subset of the IDCS patients were included in the analysis because only patients seen by the ID PA during the work week were included; therefore, these results cannot be generalized to the ID service as a whole.

The present study demonstrates that a PA can be successfully integrated into a specialty service with a complex patient population. This is a unique role for a PA in Canada and, therefore, it is unclear whether these results can be generalized to other inpatient medicine subspecialities employing PAs. Further studies that aim to evaluate PA-related outcomes in health care efficiencies would be of significant benefit to the Canadian PA literature.

The present study demonstrated that with the addition of a PA to the IDCS, health care efficiency improved, as measured by time to consult and LOS. These findings also support the objective that the introduction of PAs in Canada would provide greater access to care and potential cost savings through efficiencies achieved in the delivery of care (2).

assistants by physicians: Benefits and barriers in the Ontario health care system. Can Fam Physician 2013;59:e507-13.

12. Busse JW, Riva JJ, Nash JV, et al. Surgeon attitudes toward nonphysician screening of low back or low back-related leg pain patients referred for surgical assessment: A survey of Canadian spine surgeons. Spine 2013;38:E402-8.

13. Bohm ER, Dunbar M, Pitman D, Rhule C, Araneta J. Experience with physician assistants in a Canadian arthroplasty program. Can J Surg 2010;53:103-8.

14. Ducharme J, Alder RJ, Pelletier C, Murray D, Tepper J. The impact on patient flow after the integration of nurse practitioners and physician assistants in 6 Ontario emergency departments. CJEM 2009;11:455-61.

15. Sigurdson L. Meeting challenges in the delivery of surgical care. [Abstract]. Winnipeg, September 27 to 29, 2007: The Royal College of Physicians and Surgeons of Canada 2007 Annual Conference. Clin Invest Med 2007;30:S35-S6.

16. Health Force Ontario - Career Start Grant for Physician Assistant Graduates (2013). 2013. <www.healthforceontario.ca/UserFiles/file/ Floating/Program/PA/pa-career-start-info-package-2013-en.pdf> (Accessed April 7, 2014).

17. Canadian Institute of Health Information. DAD resource intensity weights and expected length of stay. Ottawa: Canadian Institute of Health Information, 2004.

18. Rosenbaum PR. Optimal matching for observational studies. J Am Stat Assoc 1989;84:1024-32.

19. Bergstralh EJ, Kosanke JL. Computerized Matching of Controls. Rochester: Section of Biostatistics, Mayo Foundation 1995. 


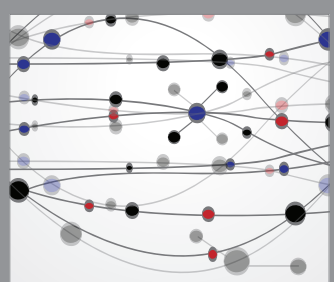

The Scientific World Journal
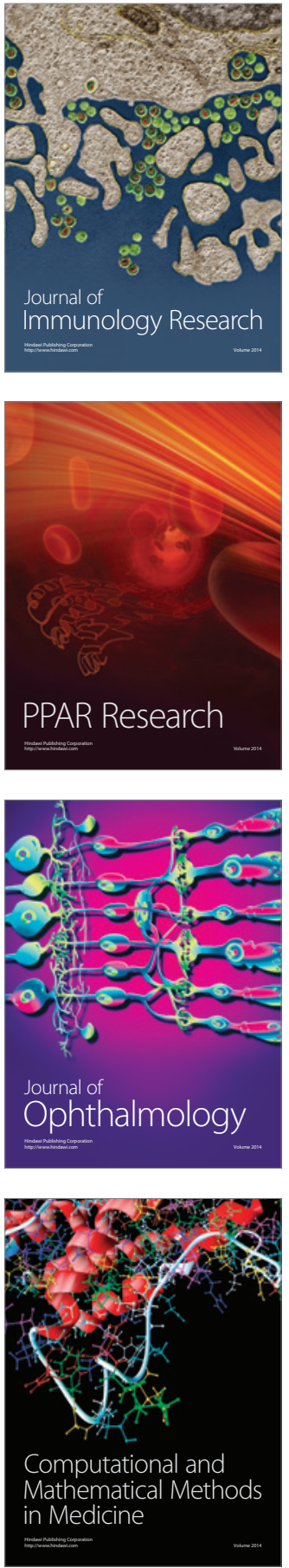

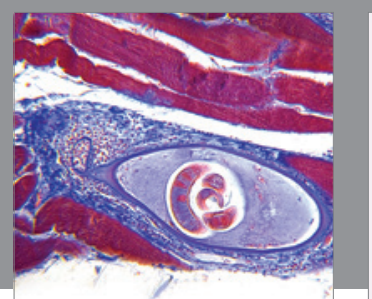

Gastroenterology Research and Practice

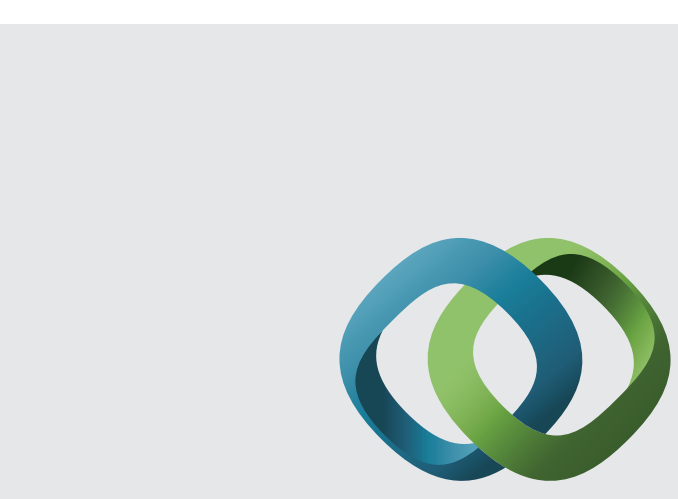

\section{Hindawi}

Submit your manuscripts at

http://www.hindawi.com
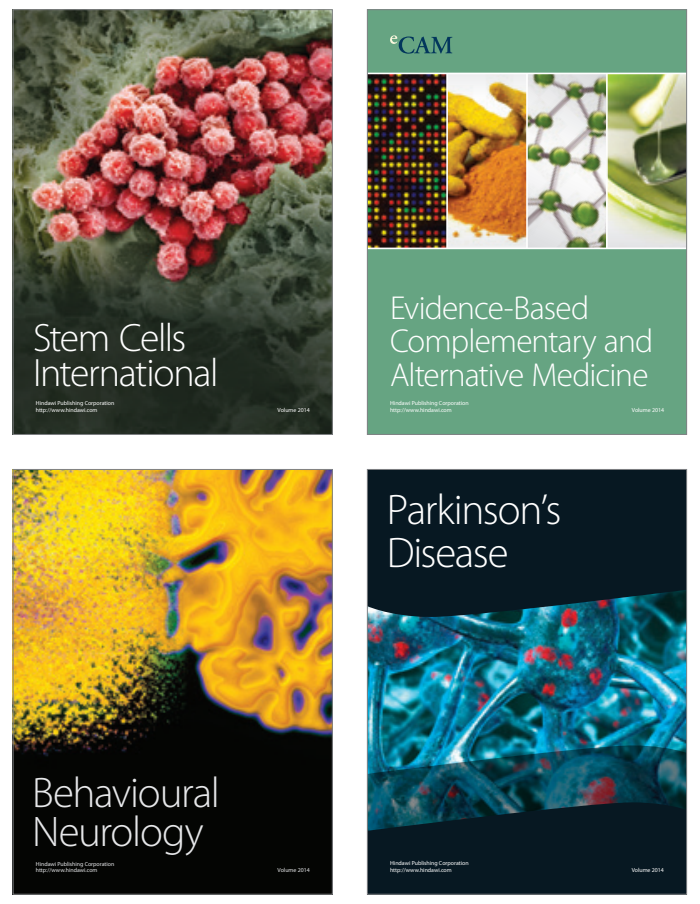
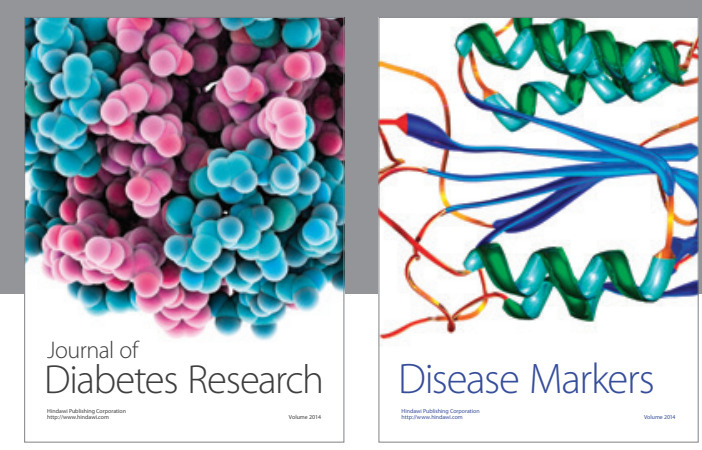

Disease Markers
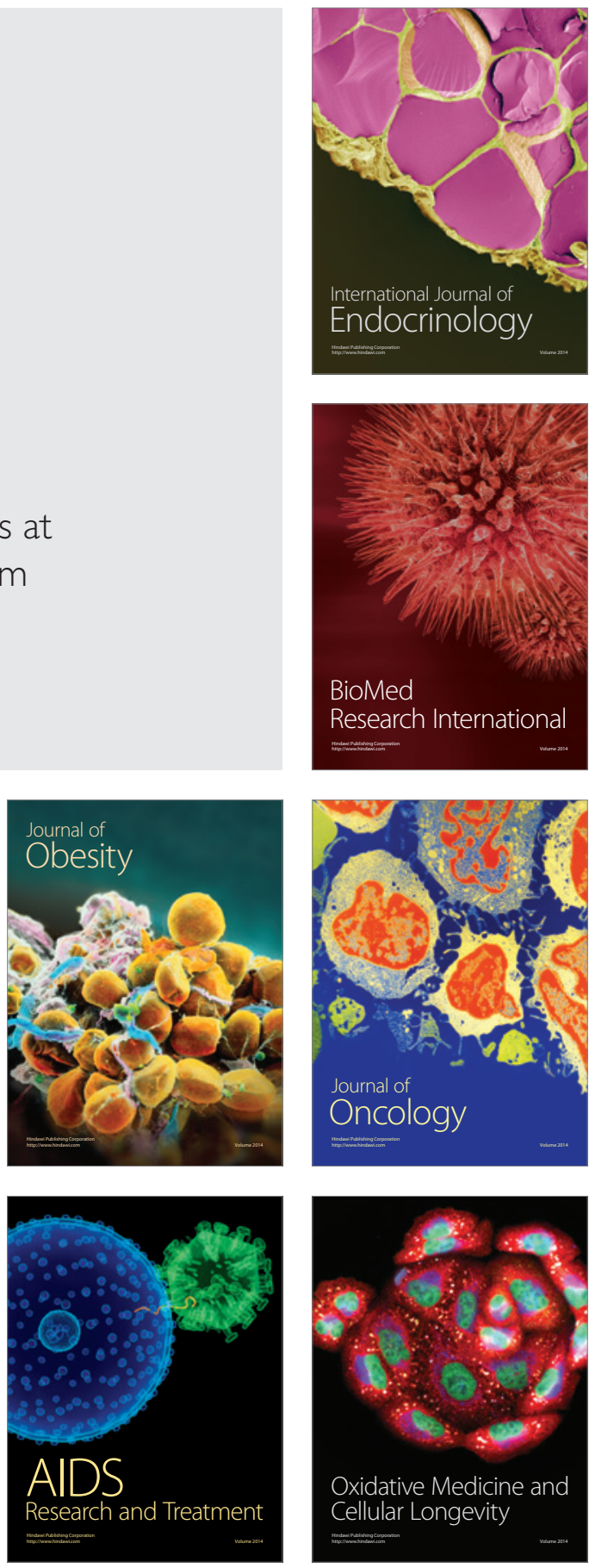